\title{
Stabilization of a purely dipolar quantum gas against collapse
}

\author{
T. KOCH, T. LAHAYE, J. METZ, B. FRÖHLICH, A. GRIESMAIER AND T. PFAU* \\ 5. Physikalisches Institut, Universität Stuttgart, Pfaffenwaldring 57, 70550 Stuttgart, Germany \\ *e-mail: t.pfau@physik.uni-stuttgart.de
}

Although the phenomenon of Bose-Einstein condensation ${ }^{1}$ is a purely statistical effect that also appears in an ideal gas, the physics of Bose-Einstein condensates (BECs) of dilute gases is considerably enriched by the presence of interactions among the atoms. In usual experiments with BECs, the only relevant interaction is the isotropic and short-range contact interaction, which is described by a single parameter, the scattering length $a$. In contrast, the dipole-dipole interaction between particles possessing an electric or magnetic dipole moment is of longrange character and anisotropic, which gives rise to new phenomena $^{2,3}$. Most prominently, the stability of a dipolar BEC depends not only on the value of the scattering length, $a$, but also strongly on the geometry of the external trapping potential ${ }^{4-7}$. Here, we report on the experimental investigation of the stability of a dipolar $\mathrm{BEC}$ of ${ }^{52} \mathrm{Cr}$ as a function of the scattering length and the trap aspect ratio. We find good agreement with a universal stability threshold arising from a simple theoretical model. Using a pancake-shaped trap with the dipoles oriented along the short axis of the trap, we are able to tune the scattering length to zero, stabilizing a purely dipolar quantum gas.

In the case of a homogeneous Bose-Einstein condensate (BEC) with pure contact interaction, the existence of a stable ground state depends on the modulus and sign of the interaction. For repulsive interaction $(a>0)$, the BEC is stable, whereas for attractive interaction $(a<0)$, the BEC is unstable. This instability can be prevented by an external trapping potential. The tendency of the gas to shrink towards the centre of the trap is in that case counteracted by the repulsive quantum pressure arising from the Heisenberg uncertainty relation. Detailed analysis ${ }^{8}$ shows that in a harmonic trap with mean frequency $\bar{\omega}$, a condensate is stable as long as the number of atoms $N$ stays below a critical value $N_{\text {crit }}$ given by

$$
N_{\text {crit }}=\frac{k a_{\mathrm{ho}}}{|a|},
$$

where $a_{\mathrm{ho}}=\sqrt{\hbar /(m \bar{\omega})}$ is the harmonic oscillator length and $k$ is a constant of the order of $1 / 2$. This scaling has been experimentally checked in ref. 9 in a BEC of ${ }^{85} \mathrm{Rb}$. The dynamics of condensates for $N \geq N_{\text {crit }}$ has been the subject of several experiments with condensates of ${ }^{7} \mathrm{Li}$ (refs 10,11) and ${ }^{85} \mathrm{Rb}$ (ref. 12). In refs 13, 14, the collapse of a Bose-Fermi mixture of ${ }^{87} \mathrm{Rb}$ and ${ }^{40} \mathrm{~K}$ was investigated. Some aspects of the dynamics such as the soliton train formation in ${ }^{7} \mathrm{Li}$ (ref. 15) and ${ }^{85} \mathrm{Rb}$ (ref. 16) remain the subject of ongoing research.

Being anisotropic and long range, the dipole-dipole interaction (DDI) differs significantly from the contact interaction, which changes the stability conditions in a system with DDI present.
Considering a purely dipolar condensate with homogeneous density polarized by an external field, it is found that owing to the anisotropy of the DDI, the BEC is unstable, independent of how small the dipole moment is ${ }^{17}$. As in the pure contact case, a trap helps to stabilize the system. In the dipolar case, however, it is not only the quantum pressure that prevents the collapse but also the anisotropy of the density distribution imprinted by the trap.

Consider a cylindrically symmetric harmonic trap

$$
V_{\text {trap }}(r, z)=\frac{1}{2} m\left(\omega_{\mathrm{r}}^{2} r^{2}+\omega_{z}^{2} z^{2}\right)
$$

with the dipoles oriented along $z$, and $r$ being the distance from the symmetry axis. As can be intuitively understood from Fig. 1a, in a pancake-shaped trap (aspect ratio $\lambda=\omega_{z} / \omega_{\mathrm{r}}>1$ ), the dipoles predominantly repel each other and the BEC is stable. In contrast, a cigar-shaped trap $(\lambda<1$, Fig. 1b) leads to mainly attractive forces and hence to a dipolar collapse. Following this simple argument, we expect that in the prolate case a positive scattering length $a$ is needed to stabilize the BEC, whereas in the oblate case, we can even afford a slightly negative $a$. The dependence of the stability of a dipolar BEC on the trap aspect ratio $\lambda$ and scattering length $a$ has been extensively studied theoretically ${ }^{4-7}$, and is experimentally investigated here.

Our measurements are carried out with a BEC of ${ }^{52} \mathrm{Cr}$ (ref. 18), which is so far the only experimentally accessible quantum gas with observable DDI ${ }^{19,20}$. To compare contact and dipolar interactions, we introduce a length scale characterizing the magnetic DDI

$$
a_{\mathrm{dd}}=\frac{\mu_{0} \mu^{2} m}{12 \pi \hbar^{2}} .
$$

The numerical prefactor in $a_{\mathrm{dd}}$ is chosen such that a homogeneous condensate becomes unstable to local density perturbations for $a \leq a_{\mathrm{dd}}$ (ref. 21). As chromium has a magnetic dipole moment of $\mu=6 \mu_{\mathrm{B}}$ ( $\mu_{\mathrm{B}}$ is the Bohr magneton), $a_{\mathrm{dd}} \simeq 15 a_{0}$, where $a_{0}$ is the Bohr radius. Far from Feshbach resonances, $a$ takes its background value $a_{\mathrm{bg}} \simeq 100 a_{0}$ (ref. 22) and the BEC is stable for any $\lambda$. To explore the unstable regime, we thus reduce the scattering length $a$, which in the vicinity of a Feshbach resonance scales as

$$
a=a_{\mathrm{bg}}\left(1-\frac{\Delta B}{B-B_{0}}\right)
$$

with the applied magnetic field $B$.

To carry out the measurements, we produce a BEC of approximately 25,000 atoms at a magnetic field that is about $10 \mathrm{G}$ above the broadest Feshbach resonance where the scattering length is approximately $85 a_{0}$ and the $\mathrm{BEC}$ is stable. Once the BEC is 

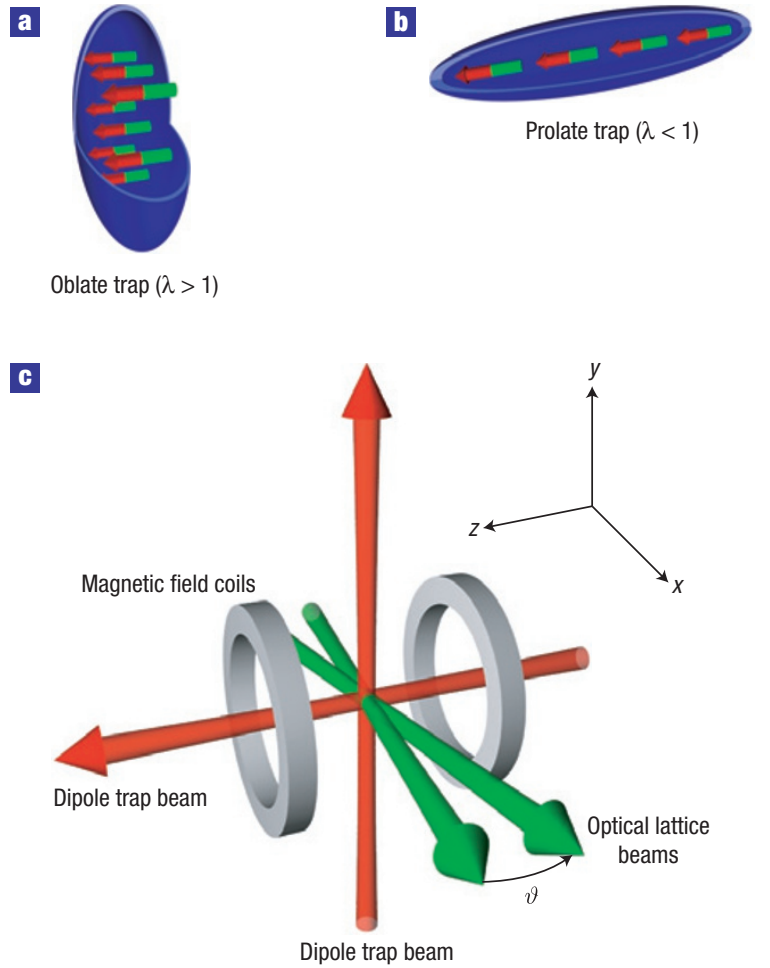

Figure 1 Intuitive picture of the trap geometry dependence of the stability of a dipolar BEC. a,b, In an oblate trap (a), the dipoles mainly repel each other, whereas in a prolate trap (b), the interaction is predominantly attractive. c, The different trapping geometries are realized by the crossed optical dipole trap (red) and a further one-dimensional optical lattice (green). The magnetic field is pointing along the symmetry axis $z$ of our traps.

obtained ${ }^{20,23}$, we adiabatically shape the trapping potential to the desired aspect ratio $\lambda$. To be able to vary $\lambda$ over a wide range, we generate the trapping potential by a crossed optical dipole trap (ODT) and a superimposed one-dimensional optical lattice along the $z$ direction (see Fig. $1 \mathrm{c}$ and Methods section).

We observe two effects when approaching the zero-crossing of the scattering length: the BEC shrinks in both directions owing to the decreasing scattering length and the ellipticity of the cloud changes as a manifestation of the enhanced dipolar effects ${ }^{20}$. Finally, when we decrease the scattering length below some critical value $a_{\text {crit }}$, the BEC atom number (determined from a bimodal fit ${ }^{24}$ of the time-of-flight absorption images) abruptly decreases (Fig. 2a,b). The disappearance of the BEC around the instability point is shown in Fig. 2c. Although slightly above $a_{\text {crit, }}$ we still see an almost pure BEC, for $a \simeq a_{\text {crit }}$ the density shows a bimodal distribution (an anisotropic, dense central peak surrounded by an isotropic gaussian cloud). Just below $a_{\text {crit }}$, the BEC collapses and the density distribution becomes a unimodal, isotropic gaussian. Note that we do not observe the formation of soliton trains as in refs 15,16 . This can be attributed to the fact that as our trap is much tighter than in those references, the initial size of our BEC is smaller than any single soliton observed in refs 15,16 .

The critical scattering length $a_{\text {crit }}$ where the condensate vanishes depends strongly on the trap aspect ratio $\lambda$. For an isotropic trap (Fig. 2a), the collapse occurs at $a \simeq 15 a_{0}$, whereas the pancakeshaped trap (Fig. 2b) can even stabilize a purely dipolar BEC $(a \simeq 0$ ). We repeated this experiment for six different traps (see Table 1), thereby covering a range of two orders of magnitude in the
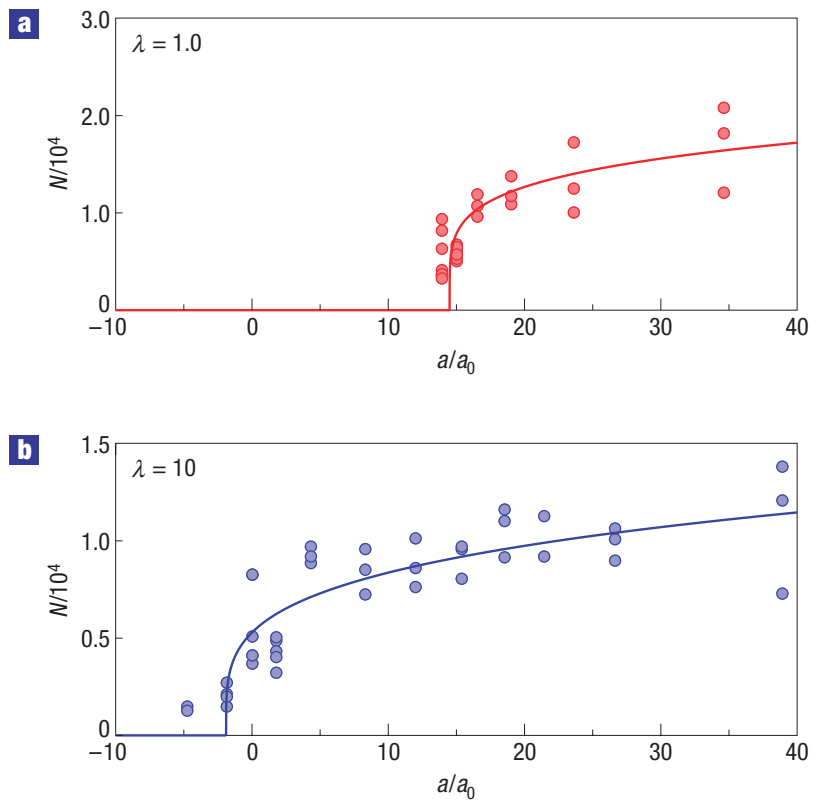

G
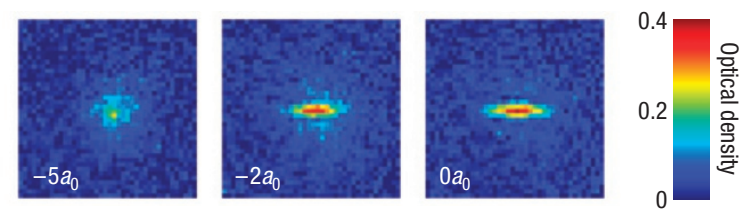

Figure 2 Decrease of the BEC atom number $\boldsymbol{N}$ around the critical scattering length $\mathbf{a}_{\text {crit }} \mathbf{a}, \mathbf{b}$, The critical point depends strongly on the aspect ratio $\lambda$ of the trap. The solid lines are fits to equation (2) used to determine the critical scattering length $a_{\text {crit }}$ (see text). c, Typical images of the atomic cloud around the critical scattering length for the trap with $\lambda=10$.

Table 1 Trap frequencies and aspect ratios of the traps used. The trap frequencies were measured by either exciting the centre-of-mass motion or parametric heating and are accurate to about $10 \%$. Traps 1-3 are provided only by the crossed optical dipole trap, whereas for traps 4-6 the horizontal dipole trap beam and the optical lattice are used.

\begin{tabular}{lcclc}
\hline Trap & $\omega_{\mathrm{r}} /(2 \pi)(\mathrm{Hz})$ & $\omega_{z} /(2 \pi)(\mathrm{Hz})$ & $\bar{\omega} /(2 \pi)(\mathrm{Hz})$ & $\lambda=\omega_{z} / \omega_{\mathrm{r}}$ \\
\hline 1 & 1,300 & 140 & 620 & 0.11 \\
2 & 890 & 250 & 580 & 0.28 \\
3 & $480^{*}$ & 480 & 480 & 1.00 \\
4 & 530 & 1,400 & 730 & 2.60 \\
5 & 400 & 2,400 & 730 & 6.00 \\
6 & 330 & 3,400 & 720 & 10.00 \\
\hline
\end{tabular}

*Trap 3 is not cylindrically symmetric (see the Methods section) and has the trap frequencies $\omega_{x}=2 \pi \times 610 \mathrm{~Hz}$ and $\omega_{y}=2 \pi \times 370 \mathrm{~Hz}$.

trap aspect ratio $\lambda$. To exclude three-body loss processes causing the abrupt decrease in the BEC atom number, we measured the lifetime of the BEC for the different traps just above $a_{\text {crit }}$ and found the same lifetime ( $\sim 10 \mathrm{~ms})$ for the different scattering lengths.

By fitting to the observed BEC atom numbers (Fig. 2a,b) the threshold function

$$
N=\max \left[0, N_{0}\left(a-a_{\text {crit }}\right)^{\beta}\right]
$$



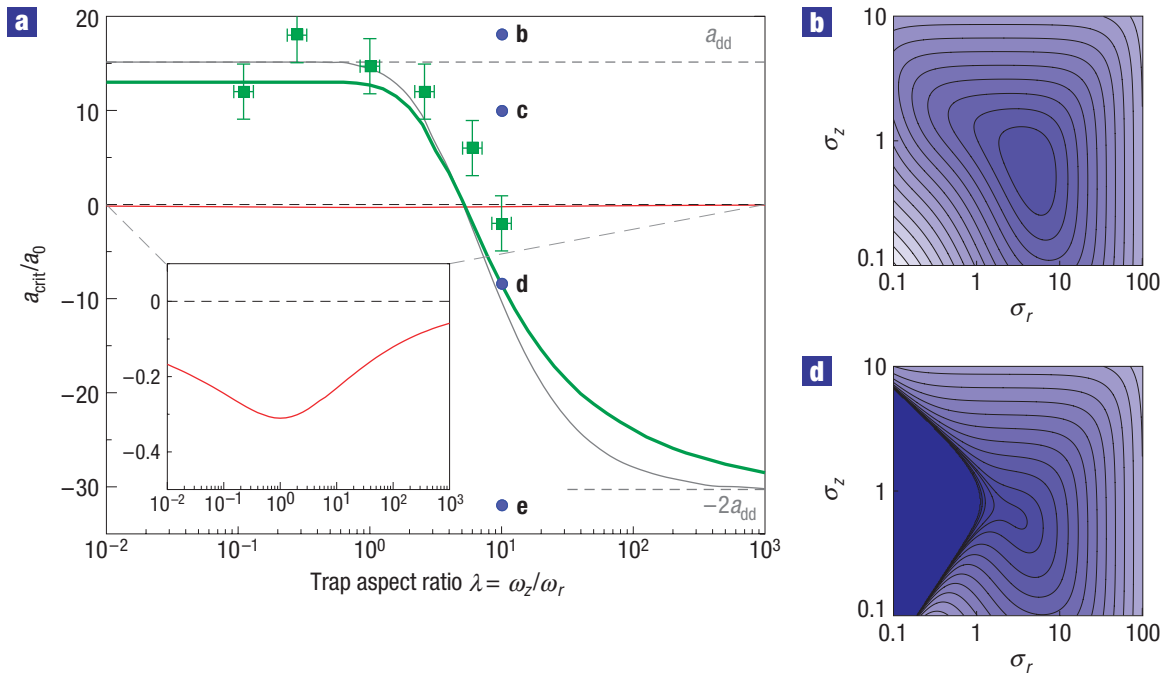
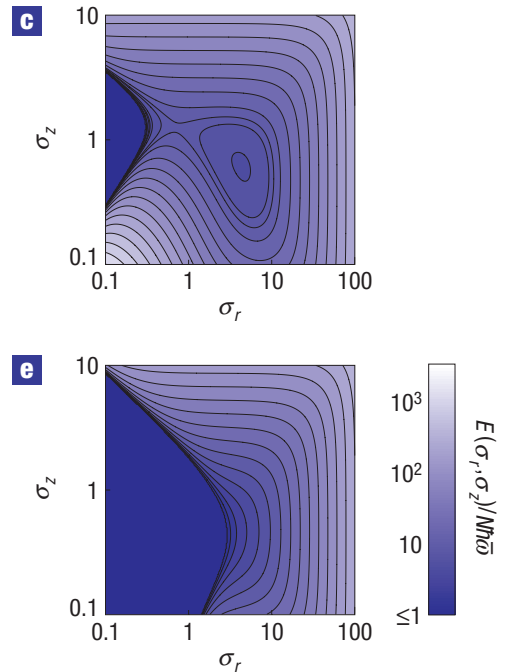

Figure 3 Stability diagram of a dipolar BEC in the $a-\lambda$ plane. a, Experimental (green squares) and theoretical (green line) values of the critical scattering length $a_{\text {crit }}$ as a function of the trap aspect ratio. The theory curve is obtained for 20,000 atoms and an average trap frequency $\bar{\omega}=2 \pi \times 700 \mathrm{~Hz}$ (the average values we find for our six traps). The red curve (magnified in the inset) marks the stability threshold for a BEC with pure contact interactions using the same parameters. The asymptotic stability boundary $\left(\mathrm{Na}_{\mathrm{dd}} / \mathrm{a}_{\mathrm{ho}} \gg 1\right)$ which for $\lambda \rightarrow 0(\lambda \rightarrow \infty)$ converges to $\mathrm{a}_{\mathrm{dd}}\left(-2 a_{\mathrm{dd}}\right)$ is plotted in grey. The error bars in $\lambda$ and $a_{\text {crit }}$ result from the uncertainty (estimated one standard deviation) in the trap frequency measurement and the calibration $a(B)$ of the scattering length. $\mathbf{b}-\mathbf{e}$, Behaviour of the energy landscape $E\left(\sigma_{r}, \sigma_{z}\right)$. Lines of equal energy are plotted for fixed $\lambda=10$ and four different values of the scattering length $a$ (blue dots in $\mathbf{a}$ ). For $a_{\text {crit }}<a<a_{\text {dd }}$ (c) the collapsed prolate ground state emerges $\left(\sigma_{r} \rightarrow 0\right.$ at finite $\left.\sigma_{z}\right)$ and the BEC becomes metastable.

where $N_{0}, a_{\text {crit }}$ and $\beta$ are fitting parameters, we find the critical scattering length $a_{\text {crit }}$. The simple functional form (2) was empirically chosen as it accounts for the fast decreasing BEC atom number at $a \simeq a_{\text {crit }}$ and for the slow decrease for $a \gg a_{\text {crit }}$. The exponent $\beta$ describing the steepness of the collapse was found to be $\beta \simeq 0.2$ for all traps. The values of $a_{\text {crit }}$ that are plotted in Fig. 3a mark the experimentally obtained stability threshold of a ${ }^{52} \mathrm{Cr}$ BEC in the plane $(\lambda, a)$. We observe a clear shift towards smaller $a$ as $\lambda$ increases. For the most oblate trap $(\lambda=10)$, we can reduce the scattering length to zero and hence access the purely dipolar regime experimentally.

To get a more quantitative insight into the collapse threshold $a_{\text {crit }}(\lambda)$, we numerically determine the critical scattering length (green curve in Fig. 3a). For this, we use a variational method to minimize the Gross-Pitaevskii energy functional ${ }^{1}$

$$
\begin{aligned}
E[\Phi]= & \int\left[\frac{\hbar^{2}}{2 m}|\nabla \Phi|^{2}+V_{\text {trap }}|\Phi|^{2}+\frac{2 \pi \hbar^{2} a}{m}|\Phi|^{4}\right. \\
& \left.+\frac{1}{2}|\Phi|^{2} \int U_{\mathrm{dd}}\left(\mathbf{r}-\mathbf{r}^{\prime}\right)\left|\Phi\left(\mathbf{r}^{\prime}\right)\right|^{2} \mathrm{~d} \mathbf{r}^{\prime}\right] \mathrm{d} \mathbf{r},
\end{aligned}
$$

where

$$
U_{\mathrm{dd}}(\mathbf{r})=\frac{\mu_{0} \mu^{2}}{4 \pi} \frac{1-3 \cos ^{2} \theta}{|\mathbf{r}|^{3}}
$$

is the interaction energy of two aligned magnetic dipoles $\mu$, with $\mathbf{r}$ being the relative position of the dipoles and $\theta$ the angle between $\mathbf{r}$ and the direction $z$ of polarization.

Similar to the work presented in refs 4-6 and 25, we use a cylindrically symmetric gaussian ansatz to evaluate the energy functional (3) with the radial and axial widths $\sigma_{r}$ and $\sigma_{z}$ as variational parameters (see the Methods section). To obtain $a_{\text {crit }}$, we lower the scattering length until the energy landscape $E\left(\sigma_{r}, \sigma_{z}\right)$ does not contain any minimum for finite $\sigma_{r}$ and $\sigma_{z}$ any more (Fig. 3b-e). Starting with large values $a>a_{\mathrm{dd}}$, we find that $E\left(\sigma_{r}, \sigma_{z}\right)$ supports a global minimum for finite $\sigma_{r}$ and $\sigma_{z}$ independently of $\lambda$ and thus the BEC is stable (Fig. 3b). Going below $a \sim a_{\mathrm{dd}}$, the absolute ground state is a collapsed infinitely thin cigar-shaped BEC $\left(\sigma_{r} \rightarrow 0\right)$ and the possible existence of a further local minimum (corresponding to a metastable state) is determined by the trap aspect ratio $\lambda$ (see Fig. $3 \mathrm{c}$, where $a_{\mathrm{dd}}>a>a_{\text {crit }}$ and Fig. $3 \mathrm{~d}$, where $a=a_{\text {crit }}$ ). Finally, below $a \sim-2 a_{\mathrm{dd}}$ (Fig. 3e), the local minimum vanishes for any $\lambda$ and the BEC is always unstable $e^{6,7}$.

In spite of the simplicity of our model, we find good agreement between experiment and theory (Fig. 3a). We checked that the different atom numbers and mean trap frequencies that we find for the six traps modify the green curve by much less than the error bars.

The behaviour of the critical scattering length $a_{\text {crit }}$ as a function of the aspect ratio $\lambda$ can be understood considering the limit $N a_{\mathrm{dd}} / a_{\mathrm{ho}} \gg 1$, which is satisfied by our average experimental value of $N a_{\mathrm{dd}} / a_{\mathrm{ho}} \simeq 23$. Owing to their linear $N$-scaling, the kinetic energy and the potential energy (equations (5) and (6) in the Methods section) can be neglected and the total energy $E\left(\sigma_{r}, \sigma_{z}\right)$ is dominated by the interaction term

$$
\left[E_{\text {contact }}+E_{\mathrm{DDI}}\right] \propto N^{2}\left[\frac{a}{a_{\mathrm{dd}}}-f(\kappa)\right] .
$$

The function $f$ (ref. 25) of the cloud aspect ratio $\kappa=\sigma_{r} / \sigma_{z}$ arises from the DDI and is discussed in the Methods section.

In this regime, where the stability is solely governed by the competition between the contact and DDI, the critical scattering length (grey curve in Fig. 3a) is implicitly given by

$$
a_{\text {crit }}(\lambda)=a_{\mathrm{dd}} f(\kappa(\lambda)) \text {. }
$$

The asymptotic behaviour of the theory curve now becomes apparent: an extremely prolate (oblate) trap forces the cloud shape to also be extremely prolate (oblate) and $f$ takes its extremal 
value $1(-2)$. Hence, we find the asymptotic values $a_{\text {crit }}=a_{\mathrm{dd}}$ for $\lambda \rightarrow 0$ and $a_{\text {crit }}=-2 a_{\mathrm{dd}}$ for $\lambda \rightarrow \infty$. Another particular point is $a_{\text {crit }}=0$, marking the aspect ratio $\lambda_{c}$ needed to stabilize a purely dipolar BEC. More precisely, as $f(1)=0$, we search for the trap in which the ground state of a purely dipolar BEC is isotropic. As the DDI tends to elongate the BEC along the $z$ direction and shrink it radially ${ }^{26}$, it is clear that the desired trap is oblate. Using our model we obtain the criterion $\lambda>\lambda_{\mathrm{c}} \approx 5.2$ for a purely dipolar BEC to be stable, a result that agrees well with the values found in refs $2,4-7$.

The grey curve in Fig. 3a that we obtain by numerically solving equation (4) shows a universal behaviour in the sense that in the large- $N$ limit $a_{\text {crit }}(\lambda)$ does not depend anymore on the absolute values of the trap frequencies and $N$. This fact clearly distinguishes the dipolar collapse from the pure contact case (red curve in Fig. 3a), where the $\lambda$-dependence, which is already weak for finite $N$ (ref. 27), completely vanishes in the limit of large $N$ as the stability criterion reads $a_{\text {crit }}(\lambda)=0$ (see equation (1) and red curve in Fig. 3a). Furthermore, the instability threshold obtained here applies for any dipolar system in a harmonic potential, such as, for example, heteronuclear molecules, where the only difference is the specific value of $a_{\mathrm{dd}}$.

In summary, we experimentally mapped the stability diagram of a dipolar BEC. The dependence on scattering length and trap aspect ratio agrees well with a simple model based on the minimization of the energy of a gaussian ansatz. By using a pancake-shaped trap, we were able to enter the regime of purely dipolar quantum gases. Although the lifetime of the purely dipolar BEC is relatively short $(\sim 10 \mathrm{~ms})$, this work opens up the route to new and exciting physics ${ }^{2}$. A clear subject for future studies is the dynamics of the dipolar collapse, which might show anisotropic features. Another remarkable property predicted to appear in a dipolar BEC contained in a pancake-shaped trap is the existence of a roton minimum in its Bogoliubov spectrum ${ }^{21}$. Furthermore, close to the collapse threshold, the existence of structured ground states is predicted ${ }^{28,29}$, a precursor for the supersolid phase ${ }^{30}$ that is expected to appear in dipolar BECs in three-dimensional optical lattices. Finally, a field that has gained increasing interest in the recent past is the study of unusual vortex lattice patterns in rotating dipolar $\mathrm{BECs}^{31,32}$.

\section{METHODS}

\section{EXPERIMENTAL SET-UP AND PROCEDURE}

To be able to vary $\lambda$ over a wide range, the trapping potential is generated by a crossed ODT $^{20,23}$ and a further optical lattice (Fig. 1c). The two lattice beams (wavelength $\lambda_{\text {latt }}=1,064 \mathrm{~nm}$, waist $w_{\text {latt }}=110 \mu \mathrm{m}$, maximum power per beam $P_{\text {latt }}=5 \mathrm{~W}$ ) propagate in the $x-z$ plane under a small angle of $\vartheta / 2=4^{\circ}$ with respect to the $x$ axis. This configuration creates a standing wave along the $z$ axis with a spacing $d=\lambda_{\text {latt }} /[2 \sin (\vartheta / 2)]=7.6 \mu \mathrm{m}$. Owing to the large spacing of the lattice and the small size of the BEC (Thomas-Fermi diameter $2 R_{z}^{\mathrm{TF}} \simeq 6 \mu \mathrm{m}$ ), we load at most two sites when ramping up the optical lattice. The resulting change in the atom number (at most dividing by 2) changes the theoretical value of $a_{\text {crit }}$ by less than $a_{0}$, which is below the size of our experimental error bars. Tunnelling processes are completely negligible on the timescale of our experiments.

By varying the powers in the beams, we are able to provide nearly cylindrically symmetric traps, with aspect ratios $\lambda$ between $\sim 1 / 10$ and $\sim 10$, while keeping the average trap frequency $\bar{\omega}=\left(\omega_{\mathrm{r}}^{2} \omega_{z}\right)^{1 / 3}$ approximately constant. More extreme aspect ratios are not used as for extreme oblate (prolate) traps the radial (axial) confinement becomes too weak to hold the atoms against gravity and remaining magnetic field gradients. The properties of the six traps that were used in the experiment are listed in Table 1. The cylindrical symmetry of the oblate traps in the $x-y$ direction is given by the fact that $\omega_{x}$ and $\omega_{y}$ are mainly determined by the horizontal ODT beam, whereas $\omega_{z}$ is given by the lattice and the vertical beam is not used. For prolate traps, $\omega_{x}$ and $\omega_{y}$ are again mainly given by the horizontal ODT beam, whereas the vertical beam determines $\omega_{z}$ and the lattice is not used. For all traps, except trap 3, we find $0.94<\omega_{x} / \omega_{y}<1.04$. For trap 3, the critical scattering length $a_{\text {crit }}$ has been calculated using a gaussian ansatz with the three widths $\sigma_{x, y, z}$ as variational parameters. The obtained value deviates by less than $a_{0}$ from the value found with the cylindrically symmetric ansatz.

After the BEC is obtained by forced evaporation in the ODT, the trapping potential is shaped in $25 \mathrm{~ms}$ to the desired aspect ratio $\lambda$. We then ramp the magnetic field within $10 \mathrm{~ms}$ to adjust the value of the scattering length. To tune $a$ accurately, we use the broadest of the resonances in ${ }^{52} \mathrm{Cr}$ (ref. 22), which is located at $B_{0} \simeq 589 \mathrm{G}$ and has a width of $\Delta B \simeq 1.5 \mathrm{G}$ (ref. 20). The current providing the magnetic field is actively stabilized with a remaining noise of less than $1 \times 10^{-5}$ r.m.s., which results in a resolution of $\Delta a \sim a_{0}$ around the zero crossing of the scattering length. After a further holding time of $2 \mathrm{~ms}$, we finally switch off the trap and take an absorption image along the $x$ axis, after a time-of-flight of $5 \mathrm{~ms}$. The BEC atom number $N$ and radii $R_{y, z}$ are obtained by fitting the density profile using a bimodal distribution ${ }^{23}$. The calibration between scattering length and magnetic field is done by solving the hydrodynamic equations for the scattering length $a$ with known $N$ and $R_{y, z}$ (refs 20,26).

\section{CALCULATION OF THE CRITICAL SCATTERING LENGTH}

To obtain an estimate of $a_{\text {crit }}$, we calculate the energy $E\left(\sigma_{r}, \sigma_{z}\right)$ (3) of the cylindrically symmetric gaussian wave function ${ }^{4-6,25}$

$$
\Phi(r, z)=\left(\frac{N}{\pi^{3 / 2} \sigma_{r}^{2} \sigma_{z} a_{\mathrm{ho}}^{3}}\right)^{1 / 2} \exp \left(-\frac{1}{2 a_{\mathrm{ho}}^{2}}\left(\frac{r^{2}}{\sigma_{r}^{2}}+\frac{z^{2}}{\sigma_{z}^{2}}\right)\right)
$$

with $\sigma_{r}$ and $\sigma_{z}$ as variational parameters. Using this ansatz, where $a_{\mathrm{ho}}=\sqrt{\hbar /(m \bar{\omega})}$, the contributions to the total energy are ${ }^{5,6}$ the zero-point fluctuations

$$
\frac{E_{\mathrm{kin}}}{\hbar \bar{\omega}}=\frac{N}{4}\left(\frac{2}{\sigma_{r}^{2}}+\frac{1}{\sigma_{z}^{2}}\right)
$$

the potential energy

$$
\frac{E_{\mathrm{pot}}}{\hbar \bar{\omega}}=\frac{N}{4 \lambda^{2 / 3}}\left(2 \sigma_{r}^{2}+\lambda^{2} \sigma_{z}^{2}\right)
$$

and the mean-field interaction energy

$$
\frac{E_{\text {contact }}+E_{\mathrm{DDI}}}{\hbar \bar{\omega}}=\frac{N^{2} a_{\mathrm{dd}}}{\sqrt{2 \pi} a_{\mathrm{ho}}} \frac{1}{\sigma_{r}^{2} \sigma_{z}}\left(\frac{a}{a_{\mathrm{dd}}}-f(\kappa)\right),
$$

with

$$
f(\kappa)=\frac{1+2 \kappa^{2}}{1-\kappa^{2}}-\frac{3 \kappa^{2} \operatorname{artanh} \sqrt{1-\kappa^{2}}}{\left(1-\kappa^{2}\right)^{3 / 2}} .
$$

The function $f(\kappa)$ is a monotonically decreasing function of the condensate aspect ratio $\kappa=\sigma_{r} / \sigma_{z}$ with the asymptotic values $f(0)=1$ and $f(\infty)=-2$, arising from the non-local term in equation (3) (ref. 25). It vanishes for $\kappa=1$, implying that for an isotropic density distribution the magnetic DDI does not contribute to the total energy. As the interaction term (7) scales as $N^{2}$, it overrules the other terms in the limit $N a_{\mathrm{dd}} / a_{\mathrm{ho}} \gg 1$.

\section{Received 18 October 2007; accepted 21 January 2008; published 24 February 2008}

\section{References}

1. Dalfovo, F., Giorgini, S., Pitaevskii, L. P. \& Stringari, S. Theory of Bose-Einstein condensation in trapped gases. Rev. Mod. Phys. 71, 463-512 (1999).

2. Baranov, M., Dobrek, L., Góral, K., Santos, L. \& Lewenstein, M. Ultracold dipolar gases—a challenge for experiments and theory. Phys. Scr. T 102, 74-81 (2002).

3. Menotti, C. \& Lewenstein, M. Ultra-cold dipolar gases. Preprint at $<$ http://arxiv.org/abs/0711.3406> (2007).

4. Santos, L., Shlyapnikov, G. V., Zoller, P. \& Lewenstein, M. Bose-Einstein condensation in trapped dipolar gases. Phys. Rev. Lett. 85, 1791-1794 (2000).

5. Góral, K. \& Santos, L. Ground state and elementary excitations of single and binary Bose-Einstein condensates of trapped dipolar gases. Phys. Rev. A 66, 023613 (2002).

6. Yi, S. \& You, L. Trapped condensates of atoms with dipole interactions. Phys. Rev. A 63 053607 (2001).

7. Eberlein, C., Giovanazzi, S. \& O'Dell, D. H. J. Exact solution of the Thomas-Fermi equation for a trapped Bose-Einstein condensate with dipole-dipole interactions. Phys. Rev. A 71, 033618 (2005).

8. Ruprecht, P. A., Holland, M. J., Burnett, K. \& Edwards, M. Time-dependent solution of the nonlinear Schrödinger equation for Bose-condensed trapped neutral atoms. Phys. Rev. A 51, 4704-4711 (1995). 9. Roberts, J. L. et al. Controlled collapse of a Bose-Einstein condensate. Phys. Rev. Lett. 86, 4211-4214 (2001)

10. Sackett, C. A., Gerton, J. M., Welling, M. \& Hulet, R. G. Measurements of collective collapse in a Bose-Einstein condensate with attractive interactions. Phys. Rev. Lett. 82, 876-879 (1999).

11. Gerton, J. M., Strekalov, D., Prodan, I. \& Hulet, R. G. Direct observation of growth and collapse of a Bose-Einstein condensate with attractive interactions. Nature 408, 692-695 (2000).

12. Donley, E. A. et al. Dynamics of collapsing and exploding Bose-Einstein condensates. Nature 412 295-299 (2001). 
13. Modugno, G. et al. Collapse of a degenerate Fermi gas. Science 297, 2240-2243 (2002).

14. Ospelkaus, C., Ospelkaus, S., Sengstock, K. \& Bongs, K. Interaction-driven dynamics of ${ }^{40} \mathrm{~K} /{ }^{87} \mathrm{Rb}$ Fermi-Bose gas mixtures in the large particle number limit. Phys. Rev. Lett. 96, 020401 (2006).

15. Strecker, K. E., Partridge, G. B., Truscott, A. G. \& Hulet, R. G. Formation and propagation of matter-wave soliton trains. Nature 417, 150-153 (2002).

16. Cornish, S. L., Thompson, S. T. \& Wieman, C. E. Formation of bright matter-wave solitons during the collapse of attractive Bose-Einstein condensates. Phys. Rev. Lett. 96, 170401 (2006).

17. Góral, K., Rzạżewski, K. \& Pfau, T. Bose-Einstein condensation with magnetic dipole-dipole forces. Phys. Rev. A 61, 051601 (2000).

18. Griesmaier, A., Werner, J., Hensler, S., Stuhler, J. \& Pfau, T. Bose-Einstein condensation of chromium. Phys. Rev. Lett. 94, 160401 (2005).

19. Stuhler, J. et al. Observation of dipole-dipole interaction in a degenerate quantum gas. Phys. Rev. Lett. 95, 150406 (2005)

20. Lahaye, T. et al. Strong dipolar effects in a quantum ferrofluid. Nature 448, 672-675 (2007).

21. Santos, L., Shlyapnikov, G. V. \& Lewenstein, M. Roton-maxon spectrum and stability of trapped dipolar Bose-Einstein condensates. Phys. Rev. Lett. 90, 250403 (2003).

22. Werner, J. et al. Observation of Feshbach resonances in an ultracold gas of ${ }^{52} \mathrm{Cr}$. Phys. Rev. Lett. 94 183201 (2005)

23. Griesmaier, A. Generation of a dipolar Bose Einstein condensate. J. Phys. B 40, R91 (2007).

24. Ketterle, W., Durfee, D. S. \& Stamper-Kurn, D. M. in Bose-Einstein Condensation in Atomic Gases (eds Inguscio, M., Stringari, S. \& Wieman, C. E.) 67-176 (Proceedings of the International School of Physics Enrico Fermi, Course CXL, IOS Press, Amsterdam, 1999).

25. Giovanazzi, S., Görlitz, A. \& Pfau, T. Ballistic expansion of a dipolar condensate. J. Opt. B 5, 208-211 (2003).

26. Giovanazzi, S. et al. Expansion dynamics of a dipolar Bose-Einstein condensate. Phys. Rev. A 74, $013621(2006)$
27. Gammal, A., Frederico, T. \& Tomio, L. Critical number of atoms for attractive Bose-Einstein condensates with cylindrically symmetrical traps. Phys. Rev. A 64, 055602 (2001)

28. Ronen, S., Bortolotti, D. C. E. \& Bohn, J. L. Radial and angular rotons in trapped dipolar gases. Phys. Rev. Lett. 98, 030406 (2007).

29. Dutta, O. \& Meystre, P. Ground-state structure and stability of dipolar condensates in anisotropic traps. Phys. Rev. A 75, 053604 (2007).

30. Góral, K., Santos, L. \& Lewenstein, M. Quantum phases of dipolar bosons in optical lattices. Phys. Rev. Lett. 88, 170406 (2002)

31. Cooper, N. R., Rezayi, E. H. \& Simon, S. H. Vortex lattices in rotating atomic Bose gases with dipolar interactions. Phys. Rev. Lett. 95, 200402 (2005).

32. Zhang, J. \& Zhai, H. Vortex lattices in planar Bose-Einstein condensates with dipolar interactions. Phys. Rev. Lett. 95, 200403 (2005).

\section{Acknowledgements}

We would like to thank L. Santos, G. V. Shlyapnikov and H.-P. Büchler for stimulating discussions and M. Fattori for his contributions in earlier stages of the experiment. We acknowledge financial support by the German Science Foundation (SFB/TR 21 and SPP 1116) and the EU (Marie-Curie fellowship contract MEIF-CT-2006-038959 to T.L.).

Correspondence and requests for materials should be addressed to T.P.

Author contributions

T.K., T.L., J.M. and B.F. carried out the experimental work and data analysis, T.K. and T.L. did the calculations on the theoretical model and A.G. and T.P. were responsible for project planning.

Reprints and permission information is available online at http://npg.nature.com/reprintsandpermissions/ 